\title{
Energy filtered imaging in a FEG-SEM for enhanced dopant contrast
}

\author{
C. Schönjahn, * C.J. Humphreys, * M.Glick** \\ *Department of Materials Science and Metallurgy, University of Cambridge, Pembroke Street, \\ Cambridge CB2 3QZ, UK \\ **Marconi Labs, Gates Building, JJ Thomson Ave, Cambridge CB3 OFD, UK
}

Two-dimensional dopant mapping using secondary electrons (SEs) in an SEM is a very useful technique for studying dopant distributions with high spatial resolution in semiconductor materials and devices. Most previous work has considered only $\mathrm{p} \quad$-type layers [1 -4$]$ and not $\mathrm{n}$-type layers presumably because the contrast for the la tter is very weak under standard imaging conditions. The purpose of this paper is to show that energy -filtered imaging of the secondary electrons in an optimized commercially available field -emission-gun SEM can substantially enhance the contrast from n-type layers. This development means that both $\mathrm{p}$-type and n-type doped regions can now be detected, imaged and mapped with high spatial resolution in an SEM, substantially increasing the utility of this technique.

A through -the-lens detector (TLD) in an FEI S -FEG XL30 was used to collect energy -filtered images. It is used in combination with an immersion lens that provides a high spatial resolution at low primary beam energies (e.g. $2 \mathrm{~nm}$ at $1 \mathrm{keV}$ ). To direct the emitted secondary electrons (SE) towards the detector (Everhart-Thornley type) an electrostatic deflection field perpendicular to the optical axis is created such that SE electrons emitted from the sample surface can reach the detector. By changing the deflector voltage $D$ one can select the maximum energy of the SEs that can reach the detector. Thus energy -filtered images may be formed in which the highest SE energy is selected by adjusting the deflector voltage. All images were acquired with a scan rate of $120 \mathrm{~ms} /$ line with 484 lines per frame. An objective aperture of $30 \mu \mathrm{m}$ was used and the working distance was $4.5 \mathrm{~mm}$. The extractor voltage of the through-the-lens detector was set to $250 \mathrm{~V}$ in order to minimize the effects of fringing fields. This high extraction voltage a lso increases the collection efficiency and energy sensitivity. A primary beam energy of $1 \mathrm{keV}$ was used. The sample studied here was MOCVD grown $\mathrm{GaN}$ on a sapphire substrate. Si-doped $\mathrm{GaN}$ (n-type) with $2 \times 10^{18} \mathrm{Si}$ atoms $/ \mathrm{cm}^{3}$ was grown on a semiinsulating GaN template nominally undoped. The GaN was cleaved along the GaN $\{11-20\}$ in order to obtain a flat surface.

Fig.1a shows the SE-image taken under standard conditions which allow almost all true SEs to reach the detector. Therefore we call this the unfiltered image. Although the n -type GaN appears slightly darker than the intrinsic material the dopant contrast is so weak that the $\mathrm{n}$-type layer is hard to distinguish from the intrinsic GaN. When the deflector voltage is reduced to $D=7 \mathrm{~V}$ only low energy SEs can reach the detector and an energy-filtered image is obtained (Fig. 1b). In that case the $n$-type $\mathrm{GaN}$ is much brighter than the $\mathrm{i}-\mathrm{GaN}$ and can be easily seen in the image. Fig.1c shows the intensity profiles that were extracted from the images described above. No te the smaller intensity in the case of the energy -filtered image compared to the unfiltered image. However, the intensity difference between n-type and intrinsic $\mathrm{GaN}$ is much larger in the energy filtered case. Because the contrast $C$ is determined by the ratio of the intensity difference between two areas and their average intensity, energy-filtered imaging can increase the contrast. From the intensity profiles we obtain $C=-0.02$ for the unfiltered image and $C>+0.09$ for the energy filtered image. Thus the $c \quad$ ontrast in the energy 
filtered image is not only enhanced but also reversed. From this observation we can conclude that an additional contrast mechanism exists under energy filtered conditions. Contrast in the unfiltered image requires a difference in th e SE-yield for $\mathrm{n}$-type and intrinsic GaN. This is not necessary to obtain contrast in energy filtered imaging conditions. For energy filtered imaging we still would see a contrast for two materials with the same SE -yield provided that the shapes of the two SE-energy distributions are different or shifted in energy with respect to each other. The latter is exactly the case for differently doped regions in a semiconductor. The energy shift arises from the built -in potential difference that exists across a junction of different doping levels in a semiconductor.

2D-dopant mapping of materials where standard imaging fails is only one application of energy filtered imaging. The technique can be successfully used for all applications in which a potential difference between materials with the same SE yield exists.

References

[1] S.L. Elliott, R, Broom, C.J. Humphreys, J. Appl. Phys., 2002 (in press)

[2] D.D. Perovic, M.R. Castell, A. Howie, C. Lavoie, T. Tiedje, J.S.W. Cole, Ultramicroscopy 58 (1995) 104

[3] D. Venables, H. Jain, D.C. Collins, J.Vac. Sci. Technol. B 16(1) (1998) 362

[4] R.Turan, D.D. Perovic, D.C. Houghton, Appl. Phys. Lett. 69 (11) (1996) 1593

[5] This research was supported by Marconi Corporation plc and FEI Electron Optics. We also like to acknowledge useful discussions with S. Mentink (Philips Electronics Netherlands).

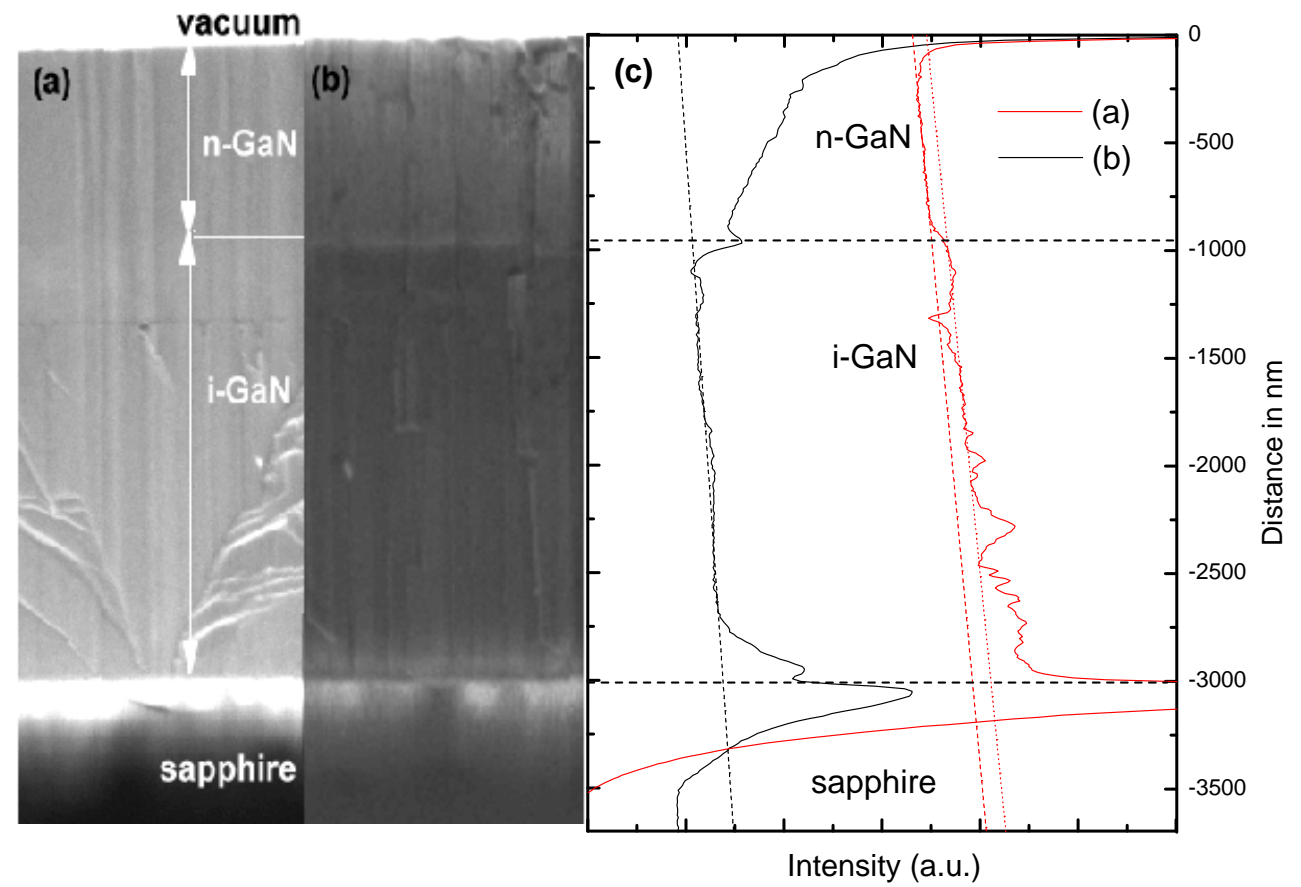

Fig. 1 SEM images of n-type GaN on i-GaN taken with TLD with (a) all SE contributing to image (unfiltered), (b) only low energy SE detected (energy filtered); (c) intensity profiles 